\title{
Neutrino Observations from the Sudbury Neutrino Observatory
}

\author{
A.W.P. Poon ${ }^{1}$ \\ Institute for Nuclear and Particle Astrophysics, Lawrence Berkeley National Laboratory, Berkeley, CA, USA
}

\begin{abstract}
.
The Sudbury Neutrino Observatory ( $\mathrm{SNO}$ ) is a water imaging Cherenkov detector. Its usage of 1000 metric tons of $\mathrm{D}_{2} \mathrm{O}$ as target allows the SNO detector to make a solar-model independent test of the neutrino oscillation hypothesis by simultaneously measuring the solar $v_{e}$ flux and the total flux of all active neutrino species. Solar neutrinos from the decay of ${ }^{8} \mathrm{~B}$ have been detected at SNO by the charged-current (CC) interaction on the deuteron and by the elastic scattering (ES) of electrons. While the CC reaction is sensitive exclusively to $v_{e}$, the ES reaction also has a small sensitivity to $v_{\mu}$ and $v_{\tau}$. In this paper, recent solar neutrino results from the SNO experiment are presented. It is demonstrated that the solar flux from ${ }^{8} \mathrm{~B}$ decay as measured from the ES reaction rate under the no-oscillation assumption is consistent with the high precision ES measurement by the Super-Kamiokande experiment. The $v_{e}$ flux deduced from the CC reaction rate in SNO differs from the Super-Kamiokande ES results by $3.3 \sigma$. This is evidence for an active neutrino component, in additional to $v_{e}$, in the solar neutrino flux. These results also allow the first experimental determination of the total active ${ }^{8} \mathrm{~B}$ neutrino flux from the Sun, and is found to be in good agreement with solar model predictions.
\end{abstract}

\section{INTRODUCTION}

For more than 30 years, solar neutrino experiments $[1,2,3,4,5,6]$ have been observing fewer neutrinos than what are predicted by the detailed models $[7,8]$ of the Sun. A comparison of the predicted and observed solar neutrino fluxes for these experiments are shown in Table 1. These experiments probe different parts of the solar neutrino energy spectrum, and show an energy dependence in the observed solar neutrino flux. These observations can be explained if the solar models are incomplete or neutrinos undergo flavor transformation while in transit to the Earth.

The Sudbury Neutrino Observatory was constructed to resolve this solar neutrino puzzle. It is capable of making simultaneous measurements of the electron-type neutrino $\left(v_{e}\right)$ flux from ${ }^{8} \mathrm{~B}$ decay in the Sun and the flux of all active

\footnotetext{
1 for the Sudbury Neutrino Observatory Collaboration: Q.R. Ahmad, R.C. Allen, T.C. Andersen, J.D. Anglin, G. Bühler, J.C. Barton, E.W. Beier, M. Bercovitch, J. Bigu, S. Biller, R.A. Black, I. Blevis, R.J. Boardman, J. Boger, E. Bonvin, M.G. Boulay, M.G. Bowler, T.J. Bowles, S.J. Brice, M.C. Browne, T.V. Bullard, T.H. Burritt, K. Cameron, J. Cameron, Y.D. Chan, M. Chen, H.H. Chen, X. Chen, M.C. Chon, B.T. Cleveland, E.T.H. Clifford, J.H.M. Cowan, D.F. Cowen, G.A. Cox, Y. Dai, X. Dai, F. Dalnoki-Veress, W.F. Davidson, P.J. Doe, G. Doucas, M.R. Dragowsky, C.A. Duba, F.A. Duncan, J. Dunmore, E.D. Earle, S.R. Elliott, H.C. Evans, G.T. Ewan, J. Farine, H. Fergani, A.P. Ferraris, R.J. Ford, M.M. Fowler, K. Frame, E.D. Frank, W. Frati, J.V. Germani, S. Gil, A. Goldschmidt, D.R. Grant, R.L. Hahn, A.L. Hallin, E.D. Hallman, A. Hamer, A.A. Hamian, R.U. Haq, C.K. Hargrove, P.J. Harvey, R. Hazama, R. Heaton, K.M. Heeger, W.J. Heintzelman, J. Heise, R.L. Helmer, J.D. Hepburn, H. Heron, J. Hewett, A. Hime, M. Howe, J.G. Hykawy, M.C.P. Isaac, P. Jagam, N.A. Jelley, C. Jillings, G. Jonkmans, J. Karn, P.T. Keener, K. Kirch, J.R. Klein, A.B. Knox, R.J. Komar, R. Kouzes, T. Kutter, C.C.M. Kyba, J. Law, I.T. Lawson, M. Lay, H.W. Lee, K.T.Lesko, J.R. Leslie, I. Levine, W. Locke, M.M. Lowry, S. Luoma, J. Lyon, S. Majerus, H.B. Mak, A.D. Marino, N. McCauley, A.B. McDonald, D.S. McDonald, K. McFarlane, G. McGregor, W. McLatchie, R. Meijer Drees, H. Mes, C. Mifflin, G.G. Miller, G. Milton, B.A. Moffat, M. Moorhead, C.W. Nally, M.S. Neubauer, F.M. Newcomer, H.S. Ng, A.J. Noble, E.B. Norman, V.M. Novikov, M. O'Neill, C.E. Okada, R.W. Ollerhead, M. Omori, J.L. Orrell, S.M. Oser, A.W.P. Poon, T.J. Radcliffe, A. Roberge, B.C. Robertson, R.G.H. Robertson, J.K. Rowley, V.L. Rusu, E. Saettler, K.K. Schaffer, A. Schuelke, M.H. Schwendener, H. Seifert, M. Shatkay, J.J. Simpson, D. Sinclair, P. Skensved, A.R. Smith, M.W.E. Smith, N. Starinsky, T.D. Steiger, R.G. Stokstad, R.S. Storey, B. Sur, R. Tafirout, N. Tagg, N.W. Tanner, R.K. Taplin, M. Thorman, P. Thornewell, P.T. Trent, Y.I. Tserkovnyak, R. Van Berg, R.G. Van de Water, C.J. Virtue, C.E. Waltham, J.-X. Wang, D.L. Wark, N. West, J.B. Wilhelmy, J.F. Wilkerson, J. Wilson, P. Wittich, J.M. Wouters, and M. Yeh
} 
TABLE 1. Summary of solar neutrino observations at different solar neutrino detectors.

\begin{tabular}{|c|c|c|c|}
\hline Experiment & Measured Flux & SSM Flux [7] & Ref. \\
\hline Homestake & $2.56 \pm 0.16$ (stat.) \pm 0.16 (sys.) SNU & $7.6_{-1.1}^{+1.3} \mathrm{SNU}$ & {$[1]$} \\
\hline SAGE & $67.2_{-7.0}^{+7.2}$ (stat.) $)_{-3.0}^{+3.5} \mathrm{SNU}$ & $128_{-7}^{+9} \mathrm{SNU}$ & [3] \\
\hline Gallex & $77.5 \pm 6.2$ (stat.) ${ }_{-4.7}^{+4.3}$ (sys.) SNU & $128_{-7}^{+9} \mathrm{SNU}$ & [4] \\
\hline GNO & $65.8_{-9.6}^{+10.2}$ (stat.) $)_{-3.6}^{+3.4}$ (sys.) SNU & $128_{-7}^{+9} \mathrm{SNU}$ & {$[5]$} \\
\hline Kamiokande & $2.80 \pm 0.19$ (stat.) \pm 0.33 (sys.) $\times 10^{6} \mathrm{~cm}^{-2} \mathrm{~s}^{-1}$ & $5.05 \times 10^{6}\left(1_{-0.16}^{+0.20}\right) \times 10^{6} \mathrm{~cm}^{-2} \mathrm{~s}^{-1}$ & {$[2]$} \\
\hline Super-Kamiokande & $2.32 \pm 0.03$ (stat. ${ }_{-0.07}^{+0.08}$ (sys.) $\times 10^{6} \mathrm{~cm}^{-2} \mathrm{~s}^{-1}$ & $5.05 \times 10^{6}\left(1_{-0.16}^{+0.20}\right) \times 10^{6} \mathrm{~cm}^{-2} \mathrm{~s}^{-1}$ & {$[6]$} \\
\hline
\end{tabular}

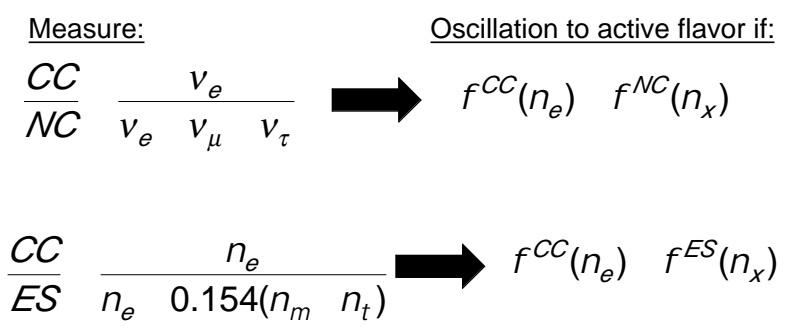

FIGURE 1. Using the measured solar neutrino fluxes from different reaction channels to provide "smoking gun" evidence of neutrino oscillation.

neutrino flavors through the following reactions:

$$
\begin{array}{lll}
v_{e}+d & \rightarrow p+p+e^{-} & (\mathrm{CC}) \\
v_{x}+d & \rightarrow p+n+v_{x} & (\mathrm{NC}) \\
v_{x}+e^{-} & \rightarrow v_{x}+e^{-} & (\mathrm{ES})
\end{array}
$$

The charged-current (CC) reaction on the deuteron is sensitive exclusively to $v_{e}$, and the neutral-current (NC) reaction has equal sensitivity to all active neutrino flavors $\left(v_{x}, x=e, \mu, \tau\right)$. Elastic scattering (ES) on electron is also sensitive to all active flavors, but with reduced sensitivity to $v_{\mu}$ and $v_{\tau}$.

Comparison of the solar neutrino flux inferred from the reaction rates of these three reaction channels under the no-oscillation assumption can provide "smoking gun" evidence for flavor-changing neutrino oscillations. If $v_{e}$ 's from the Sun transform into another active flavor, then the solar neutrino flux deduced from the CC reaction rate $\left(\phi^{\mathrm{cC}}\left(v_{e}\right)\right)$ must be less than those deduced from the ES reaction rate or the NC reaction rate. This is summarized in Figure 1.

Recent results [9] from the first measurements of the solar ${ }^{8} \mathrm{~B}$ neutrino flux by the SNO detector using the CC and ES reactions are presented in this paper. The measured $\phi^{\mathrm{ES}}\left(v_{x}\right)$ is consistent with the high precision ES measurement by the Super-Kamiokande Collaboration [6]. The measured $\phi^{\mathrm{CC}}\left(v_{e}\right)$ at SNO, however, is significantly smaller and is therefore inconsistent with the null hypothesis of a pure $v_{e}$ constituent in the solar neutrino flux. 


\section{THE SUDBURY NEUTRINO OBSERVATORY}

\section{Physical Description of the SNO Detector}

SNO [10] is an imaging water Cherenkov detector located in the Creighton \#9 mine, owned by the International Nickel Company (INCO) near Sudbury, ON, Canada. A barrel-shaped cavity with a height of 34 m and a diameter of $22 \mathrm{~m}$ was excavated at a depth of $2092 \mathrm{~m}$ (or 6000 meters of water equivalent) to house the detector. The muon flux traversing the detector is $67 \mathrm{day}^{-1}$.

Figure 2 shows a cross-sectional view of the SNO detector. The neutrino detection medium is 1000 metric tons of $99.92 \%$ isotopically pure $\mathrm{D}_{2} \mathrm{O}$ contained in a 12-m diameter acrylic sphere. The acrylic vessel is constructed out of 122 ultraviolet transmitting acrylic panels. This sphere is surrounded by 7000 metric tons of ultra-pure $\mathrm{H}_{2} \mathrm{O}$ contained in the cavity. This volume of $\mathrm{H}_{2} \mathrm{O}$ shields the detector from high energy $\gamma$ rays and neutrons originating from the cavity wall. A 17.8-m diameter stainless steel structure supports $945620-\mathrm{cm}$ inward-facing photomultiplier tubes (PMTs). A non-imaging light concentrator is mounted on each PMT, and the total photocathode coverage is 55\%. An additional 91 PMTs are mounted facing outward on the support structure to serve as cosmic veto. To cancel the vertical components of the terrestrial magnetic field, 14 horizontal magnetic compensation coils were built into the cavity wall . The maximum residual field at the PMT array is $<19 \mu \mathrm{T}$, and the reduction in photo-detection efficiency is about $2.5 \%$ from the zero-field value.

A physics event trigger is generated in the detector when there are 18 or more PMTs exceeding a threshold of $\sim 0.25$ photo-electrons within a coincidence time window of 93 ns. All the PMT hits registered in the $\sim 420$ ns window after the start of the coincidence time window are recorded in the data stream. This widened time window allows scattered and reflected Cherenkov photons to be included in the event. The mean noise rate of the PMTs is $\sim 500 \mathrm{~Hz}$, which results in $\sim 2$ noise PMT hits in this $420 \mathrm{~ns}$ window. The instantaneous trigger rate is about $15-20 \mathrm{~Hz}$, of which 6-8 Hz are physics triggers. The remaining triggers are diagnostic triggers for monitoring the well being of the detector. The trigger efficiency reaches $100 \%$ when the PMT multiplicity $\left(\mathrm{N}_{\text {hits }}\right)$ in the event window is $\geq 23$.

\section{Solar Neutrino Physics Program at SNO}

The solar neutrino physics program at SNO is designed to exploit its unique NC capability. Because the result of this NC measurement is a definitive statement on the oscillation of solar neutrinos, the SNO experiment plans to make three $\mathrm{NC}$ measurements of the total ${ }^{8} \mathrm{~B}$ active neutrino flux.

The first $\mathrm{NC}$ measurement is made with a pure $\mathrm{D}_{2} \mathrm{O}$ target. The free neutron from the NC interaction is thermalized, and in $30 \%$ of the time, a $6.25-\mathrm{MeV} \gamma$ ray is emitted following the neutron capture by deuteron. A significant portion of the $6.25-\mathrm{MeV}$ photopeak is below the neutrino analysis threshold. The second $\mathrm{NC}$ measurement is made with $\mathrm{NaCl}$ added to the $\mathrm{D}_{2} \mathrm{O}$. The free neutron is readily captured by ${ }^{35} \mathrm{Cl}$ in this detector configuration, and a cascade of $\gamma$ rays with a total energy of $8.6 \mathrm{MeV}$ follow. The neutron detection efficiency is significantly enhanced, and $\sim 45 \%$ of the NC events have a detectable signal above the analysis threshold. In the third NC measurement, discrete ${ }^{3} \mathrm{He}$ proportional counters will be installed inside the $\mathrm{D}_{2} \mathrm{O}$ volume [11]. The neutron detection efficiency of the proportional counter array is $37 \%$. In this detector configuration, the detection of the $\mathrm{CC}$ and the NC signals are decoupled, and the covariance of the CC and NC signals that appear in the first two detector configurations is eliminated in this case.

\section{SOLAR NEUTRINO ANALYSIS AT SNO}

The data presented in this paper were recorded between November 2, 1999 and January 15, 2001. The corresponding live time is 240.95 days. The target was pure $\mathrm{D}_{2} \mathrm{O}$ during this period. Figure 3 summarizes the analysis procedure. The data were divided into two sub-sets. One of these sub-sets contained $\sim 70 \%$ of the data and was used to establish the data analysis procedures. The remaining 30\% was used for a blind test of statistical bias in the analysis after the analysis procedures were settled. Analyses of the open and the blind data sets employing the same procedures show no statistically significant differences. In the following the analysis of the combined data set are presented. 


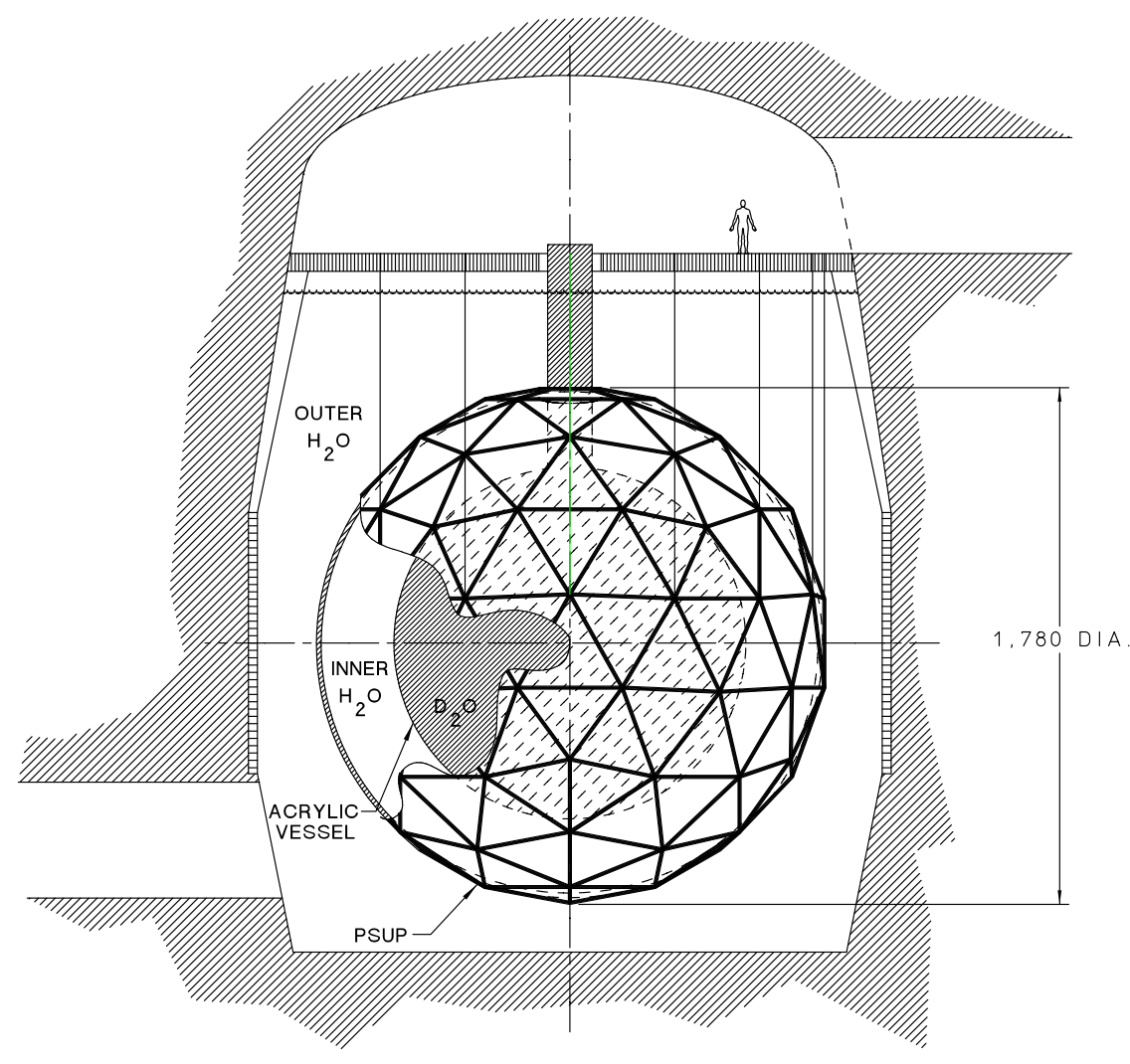

FIGURE 2. A cross-sectional view of the SNO detector. The outer geodesic structure is the PMT support ("PSUP").

\section{Pass 0 Cuts}

After removing all the detector diagnostic triggers from the data stream, a significant portion of the remaining events are instrumental backgrounds. Electrical discharges in the PMTs ("flashers") or insulating detector materials emit light. These events have characteristic PMT time and charge distributions that are significantly different from Cherenkov light, and can be eliminated using cuts based on these distributions. For example, the discharge light emitted from a flasher PMT is detected across the detector $\sim 70 \mathrm{~ns}$ after the initial discharge is registered. Some of these light-emitting instrumental backgrounds are localized near the water piping near the top of the detector. Veto PMTs were installed in this region in order to enhance the rejection efficiency of these non-Cherenkov events. Interference in the electronics system can produce false events. Most of the registered electronic channel charges in these interference events are near the pedestal, and can be removed by a cut on the mean charge of the fired PMTs. Some of these electrical discharge or electronic interference background events also have different event-to-event time correlations from physics events, and time correlation cuts are used to remove these events. Two independent instrumental background rejection schemes are used. An event-by-event comparison of the data sets reduced by these two schemes shows a difference of $<0.2 \%$.

The physics loss due to these instrumental background cuts is calibrated with a triggered ${ }^{16} \mathrm{~N} 6.13-\mathrm{MeV} \gamma$-ray source [12] and a triggered ${ }^{8} \mathrm{Li} 13-\mathrm{MeV}$ endpoint $\beta$ source [13] deployed to the $\mathrm{D}_{2} \mathrm{O}$ and $\mathrm{H}_{2} \mathrm{O}$ volumes. Further tests of the $\mathrm{N}_{\text {hits }}$ dependence in the cuts are performed with an isotropic light source at various intensities. The physics acceptance of the instrumental background cuts, weighted over the fiducial volume, is measured to be $0.9967_{-0.0008}^{+0.0018}$. Instrumental background rejection is well over $99 \%$ at this stage.

Free neutrons and high-energy $\beta$-decay nuclei from spallations induced by cosmic rays can form a significant background to the solar neutrino signal. Because the cosmic muon event rate is sufficiently low $\left(67\right.$ day $\left.^{-1}\right)$, a 20 second veto window after each cosmic muon event is employed to eliminate any contamination of the neutrino signal from the spallation products. 


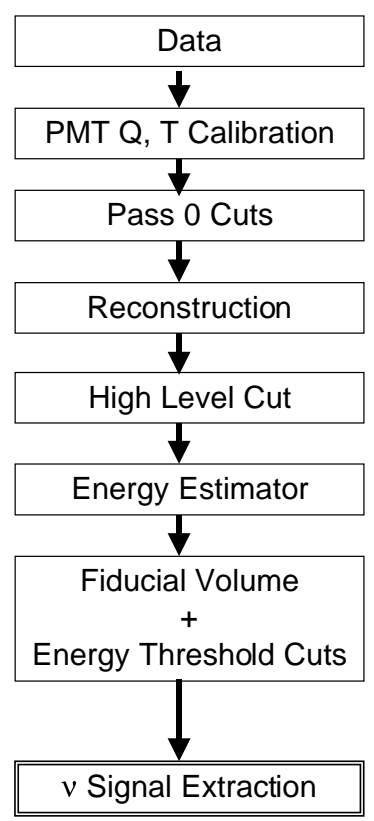

FIGURE 3. Simplified flow chart of solar neutrino analysis at SNO.

\section{Reconstruction}

After passing the instrumental background cuts, all events with $\mathrm{N}_{\text {hits }} \geq 30(\sim 3.5 \mathrm{MeV}$ electron energy) are reconstructed. The calibrated times and positions of the fired PMTs are used to reconstruct the vertex position and the direction of the particle. Two different reconstruction algorithms were developed. An event-by-event comparison shows excellent agreement between the data sets reconstructed by these two algorithms. The data presented in this paper are reconstructed using a maximum likelihood technique which uses both the time and angular characteristics of Cherenkov light. Vertex reconstruction accuracy and resolution for electrons are measured using Compton electrons from the ${ }^{16} \mathrm{~N} \gamma$-ray source, and their energy dependence is verified by the ${ }^{8} \mathrm{Li} \beta$ source. Compton scattered electrons from a $6.13-\mathrm{MeV} \gamma$ ray are preferentially scattered in the forward direction relative to the incident $\gamma$-ray direction. In order to minimize the effect of finite vertex resolution on this angular resolution measurement, only ${ }^{16} \mathrm{~N}$ events that are reconstructed to more than $150 \mathrm{~cm}$ from the source are used in the measurement. At the ${ }^{16} \mathrm{~N}$ energy ( $\sim 5.5 \mathrm{MeV}$ total electron energy), the vertex reconstruction resolution is $16 \mathrm{~cm}$ and the angular resolution is $26.7^{\circ}$. Reconstruction-related systematic uncertainties to the solar neutrino flux measurement is $\sim 4 \%$.

\section{Energy Estimator}

Figure 4 shows the relationship between the detector calibration program and event-by-event energy estimation in the analysis. Optical calibration is obtained using a near-isotropic source of pulsed laser light $[14,15]$ at 337,365 , $386,420,500$ and $620 \mathrm{~nm}$. The light source is deployed to locations accessible by the source manipulator system on two orthogonal plane in the $\mathrm{D}_{2} \mathrm{O}$, and on a linear grid in the $\mathrm{H}_{2} \mathrm{O}$. Optical parameters of different optical media in the detector are obtained at these wavelengths [16]. The attenuation lengths in $\mathrm{D}_{2} \mathrm{O}$ and $\mathrm{H}_{2} \mathrm{O}$ are found to be near the Rayleigh scattering limit. These optical parameters are inputs to the Monte Carlo/energy estimator engine.

A triggered ${ }^{16} \mathrm{~N}$ source (predominantly $6.13-\mathrm{MeV} \gamma$ ) is used to provide the absolute energy calibration. The detector energy response to the photopeak of this source provides a normalization to the PMT photon collection efficiency used in the Monte Carlo, and establish the absolute energy calibration. A long-term stability study of the detector response to the ${ }^{16} \mathrm{~N}$ source shows a linear drift of $-2.2 \pm 0.2 \%$ year $^{-1}$. The cause of this effect is under investigation, and a drift 


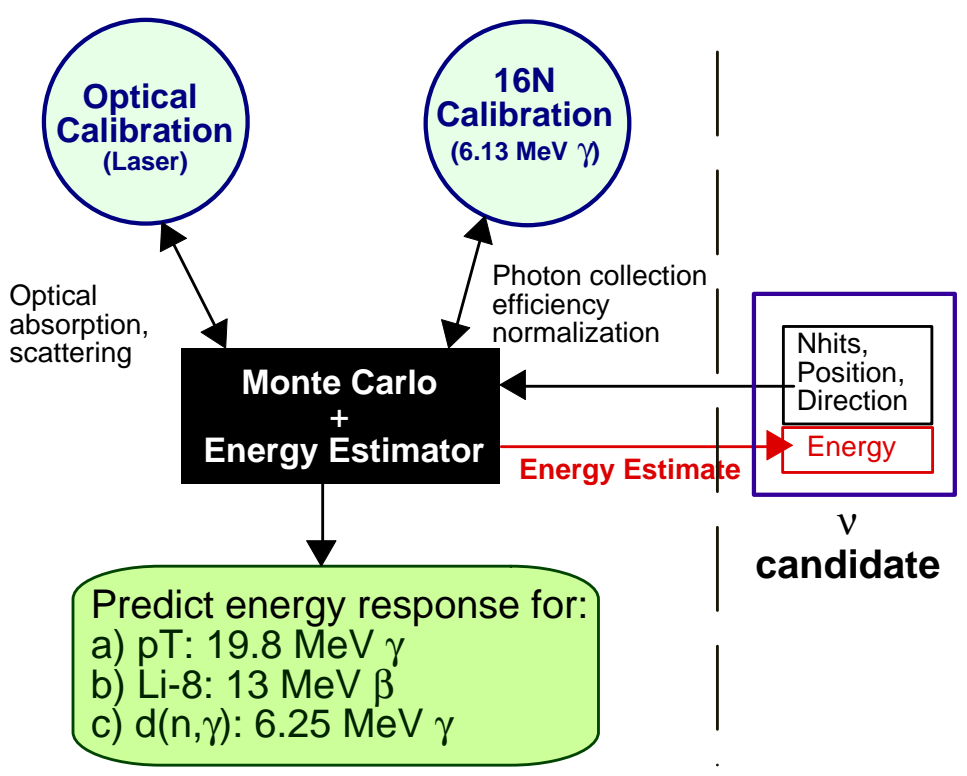

FIGURE 4. Calibration of the SNO detector and event-by-event energy estimator.

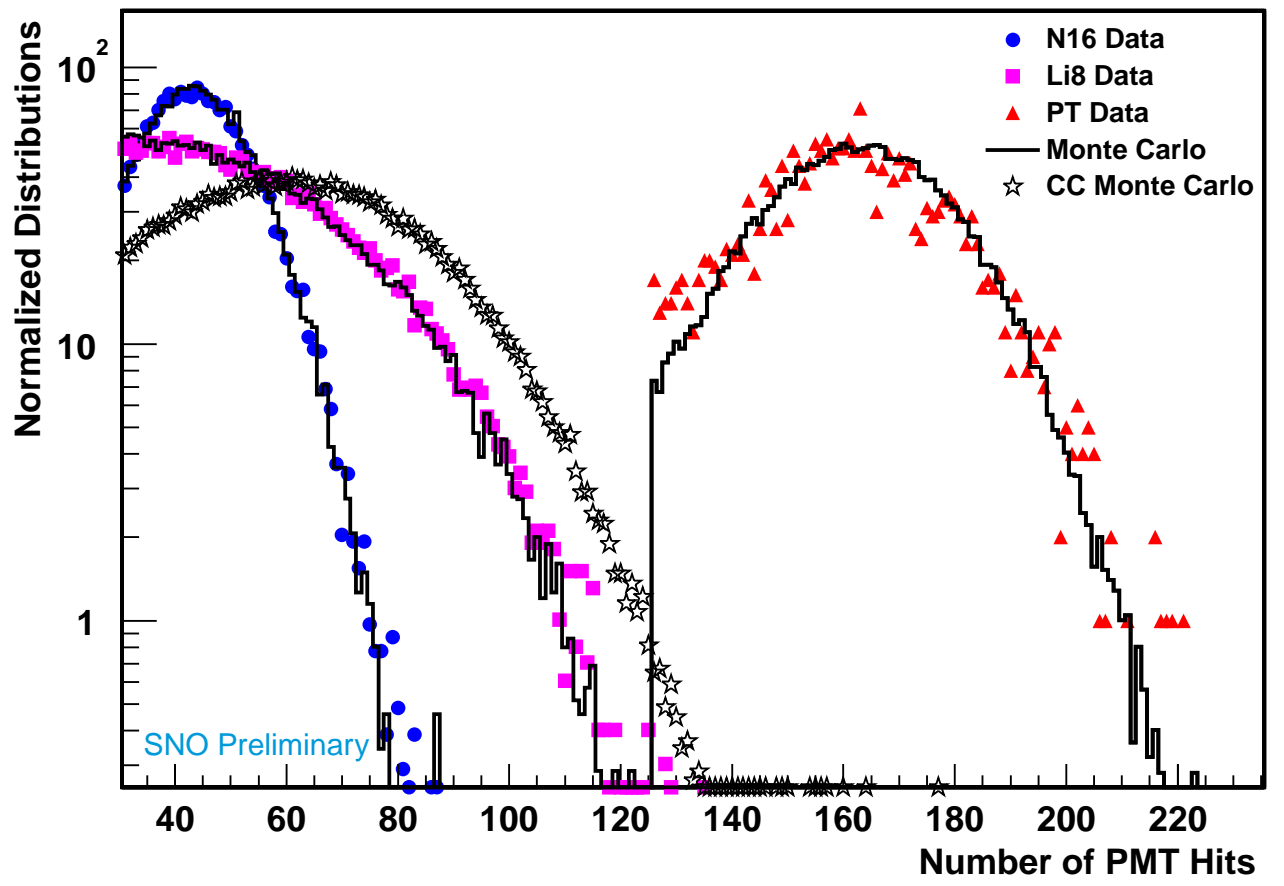

FIGURE 5. Comparison of the Monte Carlo predicted responses to different calibrated sources.

correction is applied to the event-by-event energy estimator.

This tuned Monte Carlo is then used to make predictions for the energy response to different calibration sources. The pT source generates $19.8-\mathrm{MeV} \gamma$ rays through the ${ }^{3} \mathrm{H}(\mathrm{p}, \gamma){ }^{4} \mathrm{He}$ reaction [17], and is used to check the linearity of the energy response beyond the endpoint of the ${ }^{8} \mathrm{~B}$ neutrino energy spectrum. The ${ }^{252} \mathrm{Cf}$ fission neutron source provides an extended distribution of $6.25-\mathrm{MeV} \gamma$ rays from $\mathrm{d}(\mathrm{n}, \gamma) \mathrm{t}$. Figure 5 shows a comparison of the Monte Carlo predictions and the detector responses to these sources.

The energy estimator uses the same input parameters (e.g. optical parameters) as the Monte Carlo. It assigns an 
TABLE 2. Data reduction steps

\begin{tabular}{lr}
\hline Analysis step & Number of events \\
\hline Total event triggers & 355320964 \\
Neutrino data triggers & 143756178 \\
PMT hit multiplicity $\left(\mathrm{N}_{\text {hits }}\right) \geq 30$ & 6372899 \\
Instrumental background (Pass 0) cuts & 1842491 \\
Muon followers & 1809979 \\
High level cuts & 923717 \\
Fiducial volume cut & 17884 \\
Energy threshold cut & 1169 \\
\hline Total events & $\mathbf{1 1 6 9}$ \\
\hline
\end{tabular}

effective kinetic energy $T_{\text {eff }}$ to each event based upon its position, direction and the number of hit PMTs within the prompt (unscattered) photon peak. For an electron of total energy $E_{e}$, the derived detector energy response can be parameterized by a Gaussian:

$$
R\left(E_{e f f}, E_{e}\right)=\frac{1}{\sqrt{2 \pi} \sigma_{E}\left(E_{e}\right)} \exp \left[-\frac{1}{2}\left(\frac{E_{e f f}-E_{e}}{\sigma_{E}\left(E_{e}\right)}\right)^{2}\right]
$$

where $E_{e f f}=T_{e f f}+m_{e}$, and the energy resolution is given by

$$
\sigma_{E}\left(E_{e}\right)=-0.4620+0.5470 \sqrt{E_{e}}+0.008722 E_{e} \mathrm{MeV} .
$$

The systematic uncertainty on this absolute energy calibration is found to be $\pm 1.4 \%$, which results in a neutrino flux uncertainty about 4 times larger. This is the most significant systematic uncertainty in the flux measurement. Other energy related systematic uncertainties to the flux include the energy resolution and the energy scale linearity, and each contributes to $\leq \sim 0.5 \%$ uncertainty to the flux measurement.

A second energy estimator using $\mathrm{N}_{\text {hits }}$ is employed for validation purposes. These two energy estimators give consistent results in the neutrino flux measurement.

\section{High Level Cuts}

Once the event reconstruction information becomes available after the reconstruction, several high level physics cuts are applied to the Pass 0-reduced data set to further reduce the instrumental backgrounds. These high level cuts test the hypothesis that each event has the properties of electron Cherenkov light. The reconstruction figure-of-merit cuts test for the consistency between the time and angular expectations for an event fitted to the location of the reconstructed vertex and that based on the properties of Cherenkov light and the detector response.

Two parameters are used to further characterize Cherenkov light. The average opening angle between two hit PMTs $\left(\left\langle\theta_{i j}\right\rangle\right)$, measured from the reconstructed vertex, is used to determine whether the topology of an event is consistent with Cherenkov light. The in-time ratio (ITR) is the ratio of the number of hit PMTs within an asymmetric time window around the prompt light peak to the number of calibrated PMTs in the event. Figure 6 shows the correlations between $\theta_{i j}$ and ITR for instrumental backgrounds and Cherenkov light events. As shown in the figure, this two dimensional cut has very high instrumental background rejection efficiency.

The total signal loss from the Pass 0 and the high level cuts are calibrated with the ${ }^{16} \mathrm{~N}$ and the ${ }^{8} \mathrm{Li}$ sources. For the fiducial volume (radial distance $R \leq 550 \mathrm{~cm}$ ) and the energy threshold (effective electron kinetic energy $T_{e f f} \geq 6.75 \mathrm{MeV}$ ) used in this analysis, the volume-weighted neutrino signal loss is determined to be $1.4_{-0.6}^{+0.7 \%}$.

The residual instrumental background contamination in the neutrino signal after the Pass 0 and the high level cuts is estimated by a bifurcated analysis, in which the signal contamination is obtained from cross calibrating the background leakage of two groups of orthogonal cuts. For the same fiducial volume and energy thresholds, the instrumental background contamination is estimated to be $<3$ events (95\% C.L.), or $0.2 \%$ of the final neutrino candidate data set. Table 2 summarizes the sequence of cuts that are used to reduce the raw data to 1169 neutrino candidate events. 


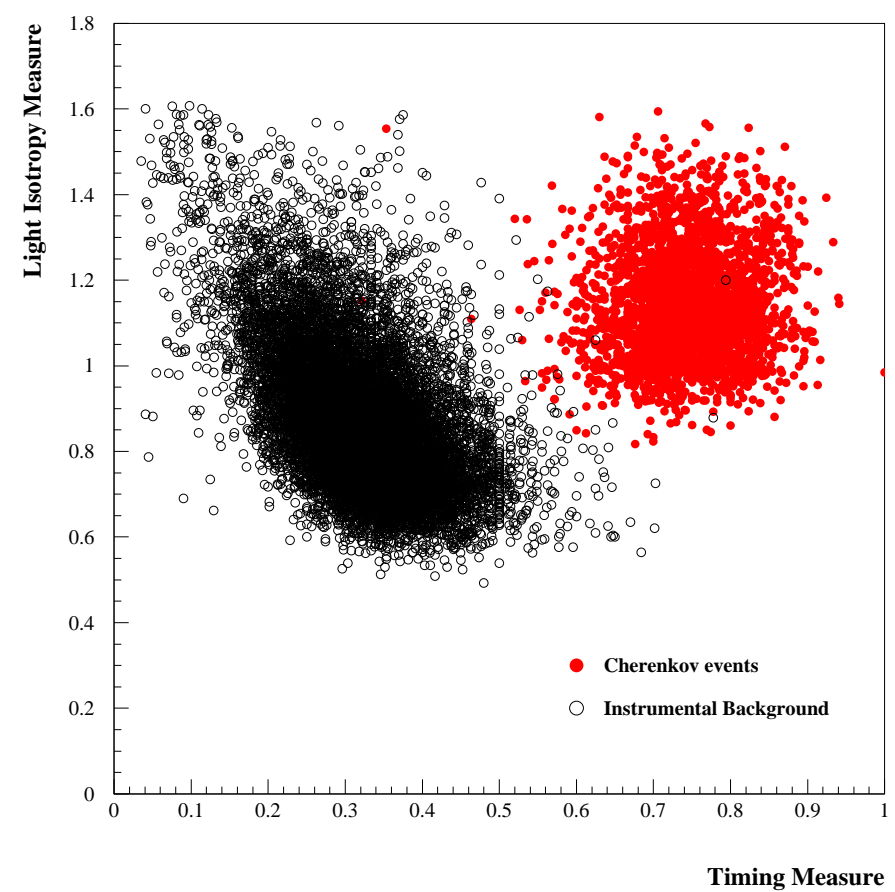

FIGURE 6. Separation of instrumental backgrounds and Cherenkov light events using the high level cuts.

\section{Physics Backgrounds}

Figure 7 shows the radial distribution of event candidates with $T_{\text {eff }} \geq 6.75 \mathrm{MeV}$ as a function of the volume-weighted radial variable $\left(R / R_{A V}\right)^{3}$, where $R_{A V}=600 \mathrm{~cm}$ is the radius of the acrylic vessel. Above this energy threshold, the neutrino signals include $\mathrm{CC}$ in the $\mathrm{D}_{2} \mathrm{O}, \mathrm{ES}$ in the $\mathrm{D}_{2} \mathrm{O}$ and $\mathrm{H}_{2} \mathrm{O}$, and the residual tail of neutron capture events (which can be $\mathrm{NC}$ or backgrounds from the photodisintegration of the deuteron), high energy tail of the internal radioactivity background, and high energy $\gamma$ rays from the cavity wall. The simulated neutrino signals, weighted by the results from the signal extraction, are shown in Figure 7. The data show a clear neutrino signal within the $\mathrm{D}_{2} \mathrm{O}$. For the $\mathrm{H}_{2} \mathrm{O}$ region $\left(\left(R / R_{A V}\right)^{3}>1\right)$, the background contribution rises until it reaches the acceptance cutoff of the PMT light concentrators at $R \sim 7 \mathrm{~m}$.

Detectable internal radioactivity signals are dominated by the $\beta \gamma$ decays of ${ }^{208} \mathrm{Tl}$ and ${ }^{214} \mathrm{Bi}$, which are daughters in the natural Th and $\mathrm{U}$ chains. These $\beta \gamma$ radionuclei can also emit $\gamma$ rays with sufficient energy to photodisintegrate the deuteron. The free neutron from this break-up is indistinguishable from the NC signal. However, this neutron background can be subtracted from the total neutron signal in the detector if the internal radioactivity level of the detector is known. In this analysis, most of the Cherenkov signals from the $\beta \gamma$ decays are removed by the high energy threshold imposed. Internal radioactivity levels in the $\mathrm{D}_{2} \mathrm{O}$ and $\mathrm{H}_{2} \mathrm{O}$ are measured by regular low level radio-assays of $\mathrm{U}$ and Th chain daughters. The light isotropy parameter $\theta_{i j}$ is also used to provide an in situ monitoring of the these backgrounds. Both techniques show that the $\mathrm{U}$ and Th radioactivity levels in the $\mathrm{D}_{2} \mathrm{O}$ and the $\mathrm{H}_{2} \mathrm{O}$ are either at or below the target levels.

There are also $\beta \gamma$ contributions from the construction materials in the PMT support structure and the PMTs to the low energy background. Monte Carlo simulations predict that these contributions are insignificant to the flux measurement. This was verified by the deployment of an encapsulated Th source in the vicinity of the PMT support structure. Contributions from all sources of low energy backgrounds to the neutrino flux measurement is $<0.2 \%$.

High energy $\gamma$ rays from $(\alpha, \gamma),(\alpha, \mathrm{n} \gamma)$ and $(\alpha, \mathrm{p} \gamma)$ reactions in the cavity wall are significantly attenuated by the $\mathrm{H}_{2} \mathrm{O}$ shield. By deploying the ${ }^{16} \mathrm{~N}$ source to the vicinity of the PMT support structure, the contribution of these $\gamma$ rays in the event candidate set is found to be $<10$ events $(68 \% \mathrm{CL}$ ), or a $1.9 \%$ uncertainty to the ES flux and a $0.8 \%$ uncertainty to the CC flux. 


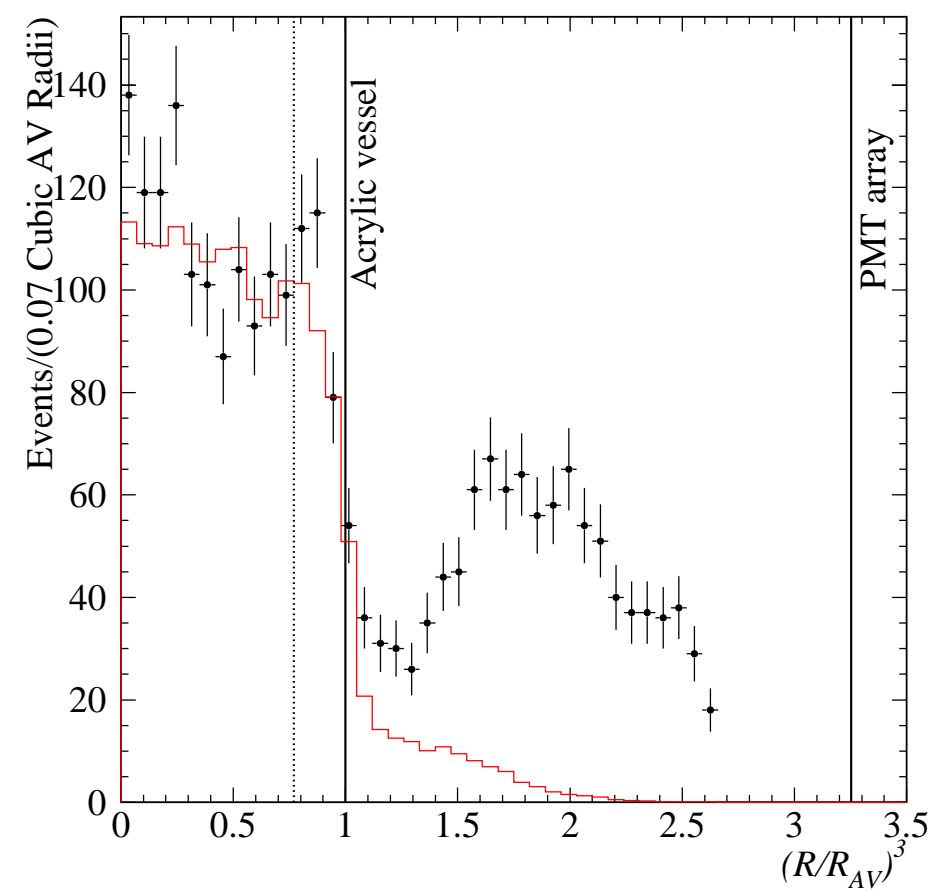

FIGURE 7. Radial distribution of event candidates with $T_{e f f} \geq 6.75 \mathrm{MeV}$ as a function of the volume-weighted radial variable $\left(R / R_{A V}\right)^{3}$. The Monte Carlo simulation of the signals, weighted by the results from the signal extraction, is shown as the histogram. The dotted line indicates the fiducial volume used in this analysis.

\section{Solar Neutrino Signal Extraction}

The extended maximum likelihood method is used in extracting the CC, ES and neutron contributions in the candidate data set. Data distributions in $T_{e f f},\left(R / R_{A V}\right)^{3}$ and $\cos \theta_{\odot}$ are simultaneously fitted to the probability density functions (PDFs) generated from Monte Carlo simulations assuming no flavor transformation and the ${ }^{8} \mathrm{~B}$ spectrum from Ortiz et al. [18] $\cos \theta_{\odot}$ is the angle between the reconstructed direction of the event and the instantaneous direction from the Sun to the Earth. This distribution is shown in Figure 8. The forward peak $\left(\cos \theta_{\odot} \sim 1\right)$ arises from the strong directionality in the ES reaction. The $\cos \theta_{\odot}$ distribution for the $\mathrm{CC}$ reaction, before accounting for the detector response, is expected to be (1-0.340 $\left.\cos \theta_{\odot}\right)$ [19]. The extraction yields $975.4 \pm 39.7 \mathrm{CC}$ events, $106.1 \pm 15.2$ ES events and $87.5 \pm 24.7$ neutron events for $T_{e f f} \geq 6.75 \mathrm{MeV}$ and $R \leq 550 \mathrm{~cm}$. The uncertainties given above are statistical only, and the systematic uncertainties are summarized in Table 3.

The ${ }^{8} \mathrm{~B}$ neutrino flux can be determined from normalizing the observed integrated event rate above the energy threshold. Assuming the ${ }^{8} \mathrm{~B}$ spectrum from Ref. [18], the flux deduced from the CC and the ES reactions are:

$$
\begin{aligned}
& \left.\phi_{\mathrm{SNO}}^{\mathrm{CC}}\left(v_{e}\right)=1.75 \pm 0.07 \text { (stat.) }{ }_{-0.11}^{+0.12} \text { (sys.) } \pm 0.05 \text { (theor.) }\right) \times 10^{6} \mathrm{~cm}^{-2} \mathrm{~s}^{-1} \\
& \phi_{\mathrm{SNO}}^{\mathrm{ES}}\left(v_{x}\right)=2.39 \pm 0.34 \text { (stat.) }{ }_{-0.14}^{+0.16} \text { (sys.) } \times 10^{6} \mathrm{~cm}^{-2} \mathrm{~s}^{-1}
\end{aligned}
$$

where the theoretical uncertainty is the CC cross section uncertainty [20]. Radiative corrections to the CC cross section have not been applied to the CC cross section, but they are expected to decrease the measured $\phi_{\mathrm{SNo}}^{\mathrm{CC}}\left(v_{e}\right)$ by up to a few percent [21]. The difference between $\phi_{\mathrm{SNO}}^{\mathrm{CC}}$ and $\phi_{\mathrm{SNO}}^{\mathrm{ES}}$ is $0.64 \pm 0.40 \times 10^{6} \mathrm{~cm}^{-2} \mathrm{~s}^{-1}$, or $1.6 \sigma$. The ratio of $\phi_{\mathrm{SNO}}^{\mathrm{CC}}$ to the predicted ${ }^{8} \mathrm{~B}$ solar neutrino flux from BPB01 solar model [7] is $0.347 \pm 0.029$ where all the uncertainties are added in quadrature. Independent analyses using $\mathrm{N}_{\text {hits }}$ as energy estimator, or in various fiducial volumes up to $620 \mathrm{~cm}$ with the inclusion of background PDFs in the signal extraction give consistent results.

The Super-Kamiokande experiment has made a high precision measurement of the ${ }^{8} \mathrm{~B}$ solar neutrino flux by the ES reaction:

$$
\left.\phi_{\mathrm{SK}}^{\mathrm{ES}}\left(v_{x}\right)=2.32 \pm 0.03 \text { (stat. }\right)_{-0.07}^{+0.08}(\mathrm{sys} .) \times 10^{6} \mathrm{~cm}^{-2} \mathrm{~s}^{-1} .
$$




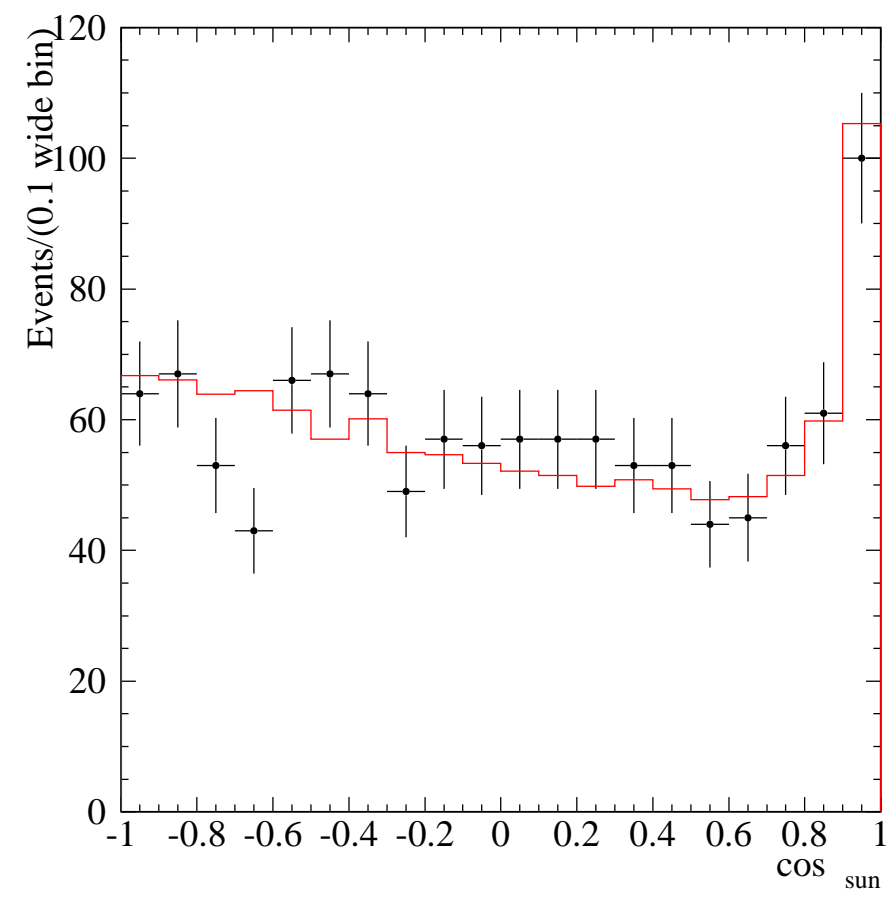

FIGURE 8. $\cos \theta_{\odot}$ distribution from the candidate event set. The Monte Carlo simulation of the signals, weighted by the results from the signal extraction, is shown as the histogram.

TABLE 3. Systematic uncertainties on fluxes

\begin{tabular}{lrr}
\hline Error source & $\begin{array}{r}\text { CC error } \\
\text { (percent) }\end{array}$ & $\begin{array}{r}\text { ES error } \\
\text { (percent) }\end{array}$ \\
\hline Energy scale & $-5.2,+6.1$ & $-3.5,+5.4$ \\
Energy resolution & \pm 0.5 & \pm 0.3 \\
Energy scale non-linearity & \pm 0.5 & \pm 0.4 \\
Vertex accuracy & \pm 3.1 & \pm 3.3 \\
Vertex resolution & \pm 0.7 & \pm 0.4 \\
Angular resolution & \pm 0.5 & \pm 2.2 \\
High energy $\gamma^{\prime}$ s & $-0.8,+0.0$ & $-1.9,+0.0$ \\
Low energy background & $-0.2,+0.0$ & $-0.2,+0.0$ \\
Instrumental background & $-0.2,+0.0$ & $-0.6,+0.0$ \\
Trigger efficiency & 0.0 & 0.0 \\
Live time & \pm 0.1 & \pm 0.1 \\
Cut acceptance & $-0.6,+0.7$ & $-0.6,+0.7$ \\
Earth orbit eccentricity & \pm 0.1 & \pm 0.1 \\
17O, ${ }^{18}$ O & 0.0 & 0.0 \\
\hline Experimental uncertainty & $\mathbf{- 6 . 2 , + 7 . 0}$ & $\mathbf{- 5 . 7 , + 6 . 8}$ \\
\hline Cross section & 3.0 & 0.5 \\
Solar Model & $-16,+20$ & $-16,+20$ \\
\hline
\end{tabular}

$\phi_{\mathrm{SNO}}^{\mathrm{ES}}\left(\nu_{x}\right)$ and $\phi_{\mathrm{SK}}^{\mathrm{ES}}\left(v_{x}\right)$ are consistent with each other. Assuming that the systematic uncertainties are normally distributed, the difference is

$$
\phi_{\mathrm{SK}}^{\mathrm{ES}}\left(v_{x}\right)-\phi_{\mathrm{SNO}}^{\mathrm{CC}}\left(v_{e}\right)=0.57 \pm 0.17 \times 10^{6} \mathrm{~cm}^{-2} \mathrm{~s}^{-1},
$$

or $3.3 \sigma$. The probability that the observed $\phi_{\mathrm{SNO}}^{\mathrm{CC}}\left(v_{e}\right)$ is a $\geq 3.3 \sigma$ downward fluctuation of $\phi_{\mathrm{SK}}^{\mathrm{ES}}\left(v_{x}\right)$ is $0.04 \%$.

If $v_{e}$ 's from ${ }^{8} \mathrm{~B}$ decays in the Sun oscillate exclusively to sterile neutrinos, the SNO CC-derived ${ }^{8} \mathrm{~B}$ flux with $T_{e f f} \geq 6.75 \mathrm{MeV}$ would be consistent with the integrated Super-Kamiokande ES-derived flux above a threshold of $8.5 \mathrm{MeV}$ [22]. The difference between these derived fluxes after adjusting for the ES threshold [6] is 

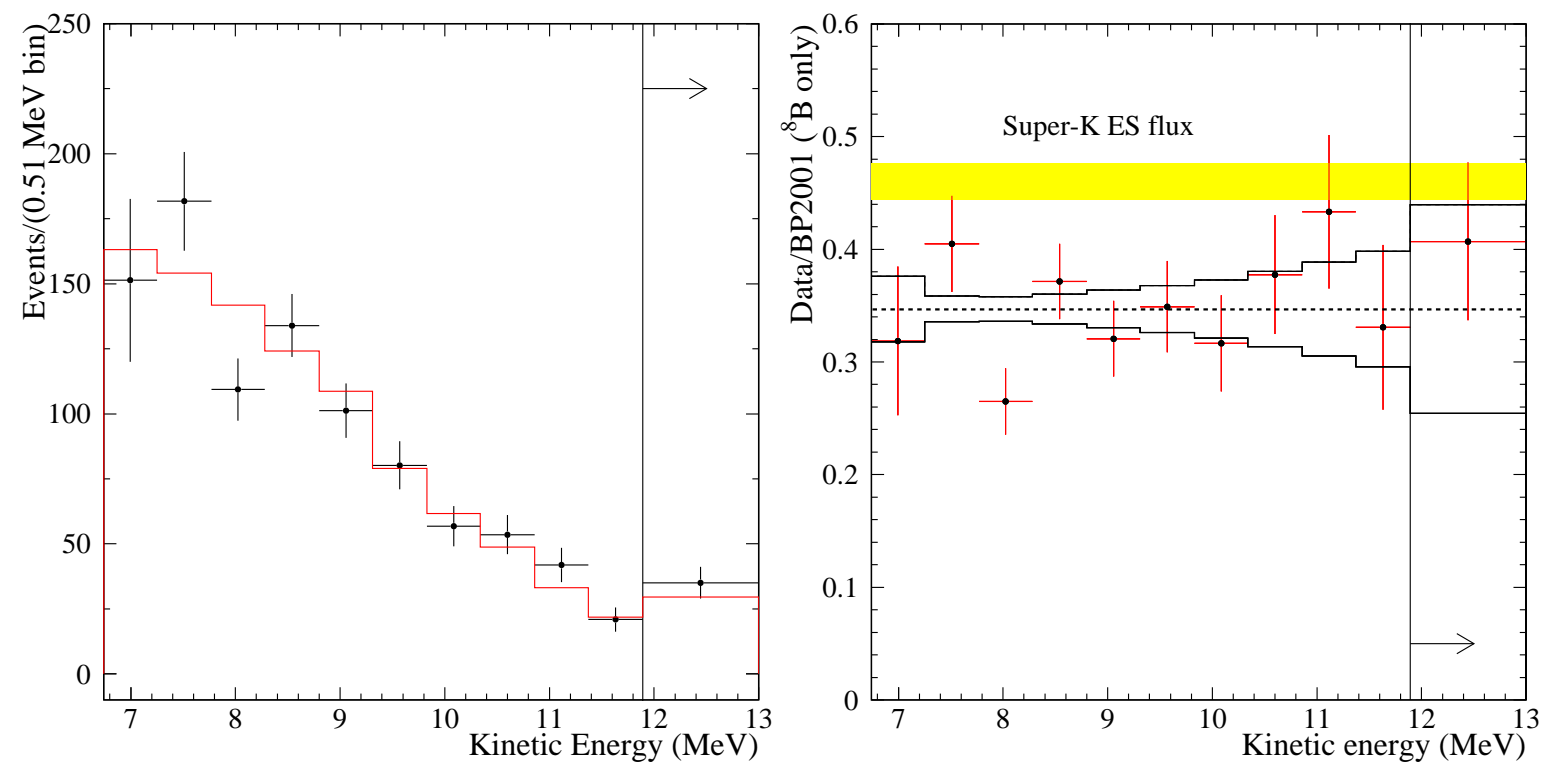

FIGURE 9. SNO CC energy spectrum. Left: The extracted CC kinetic energy spectrum from a shape-unconstrained fit of events with $R \leq 550 \mathrm{~cm}$ and $T_{e f f} \geq 6.75 \mathrm{MeV}$. The error bars are statistical only. The expected undistorted ${ }^{8} \mathrm{~B}$ spectrum, derived from Ref. [18], is shown as the histogram. Right: The ratio of the extracted CC spectrum to the expected kinetic energy spectrum. The band at each energy bin represent the $1 \sigma$ uncertainty derived from the most significant energy-dependent systematic uncertainties. The uncertainties in the ${ }^{8} \mathrm{~B}$ spectrum have not been included.

$0.53 \pm 0.17 \times 10^{6} \mathrm{~cm}^{-2} \mathrm{~s}^{-1}$, or $3.1 \sigma$. The probability of $\mathrm{a} \geq 3.1 \sigma$ downward fluctuation is $0.13 \%$. Therefore, the results presented here are evidence for a non-electron type active neutrino component in the solar neutrino flux.

The CC energy spectrum can be extracted from the data by repeating the signal extraction with the CC energy spectral constraint removed. This is shown in Figure 9. There is no evidence for spectral distortion under the nooscillation hypothesis.

\section{TOTAL ACTIVE ${ }^{8}$ B NEUTRINO FLUX}

Recall from Figure 1 that the ES reaction is sensitive to all active neutrino flavors, but with reduced sensitivity for $v_{\mu}$ and $v_{\tau}$. Using the high precision ES measurement $\phi_{\mathrm{SK}}^{\mathrm{ES}}\left(v_{x}\right)$ and the pure $v_{e}$ flux from $\phi_{\mathrm{SNo}}^{\mathrm{CC}}\left(v_{e}\right)$, one can infer the flux of non-electron flavor active neutrino $\phi\left(v_{\mu \tau}\right)$ :

$$
\phi_{\mathrm{SK}}^{\mathrm{ES}}=\phi\left(v_{e}\right)+0.154 \phi\left(v_{\mu \tau}\right) \text {. }
$$

This is shown in Figure 10, in which $\phi\left(v_{\mu \tau}\right)$ is shown against $\phi\left(v_{e}\right)$. The two data bands are $1 \sigma$ measurements of $\phi_{\mathrm{sNo}}^{\mathrm{cC}}$ and $\phi_{\mathrm{SK}}^{\mathrm{ES}}$, and the error ellipses are $68 \%, 95 \%$ and $99 \%$ joint probability contours for $\phi\left(v_{e}\right)$ and $\phi\left(v_{\mu \tau}\right)$. The best fit to $\phi\left(v_{\mu \tau}\right)$ is

$$
\phi\left(v_{\mu \tau}\right)=3.69 \pm 1.13 \times 10^{6} \mathrm{~cm}^{-2} \mathrm{~s}^{-1} .
$$

The total ${ }^{8} \mathrm{~B}$ flux derived from the SNO and the Super-Kamiokande experiments is shown as the diagonal band $\left(\phi_{x}^{\mathrm{SK}+\mathrm{SNO}}\right)$ in Figure 10. The agreement with the standard solar model prediction $\left(\phi_{x}^{\mathrm{SSM}}\right)$ is good. The total flux of of active ${ }^{8} \mathrm{~B}$ neutrinos is found to be

$$
\phi\left(v_{x}\right)=5.44 \pm 0.99 \times 10^{6} \mathrm{~cm}^{-2} \mathrm{~s}^{-1},
$$

This is the first determination of the total flux of ${ }^{8} \mathrm{~B}$ neutrinos generated by the Sun. 


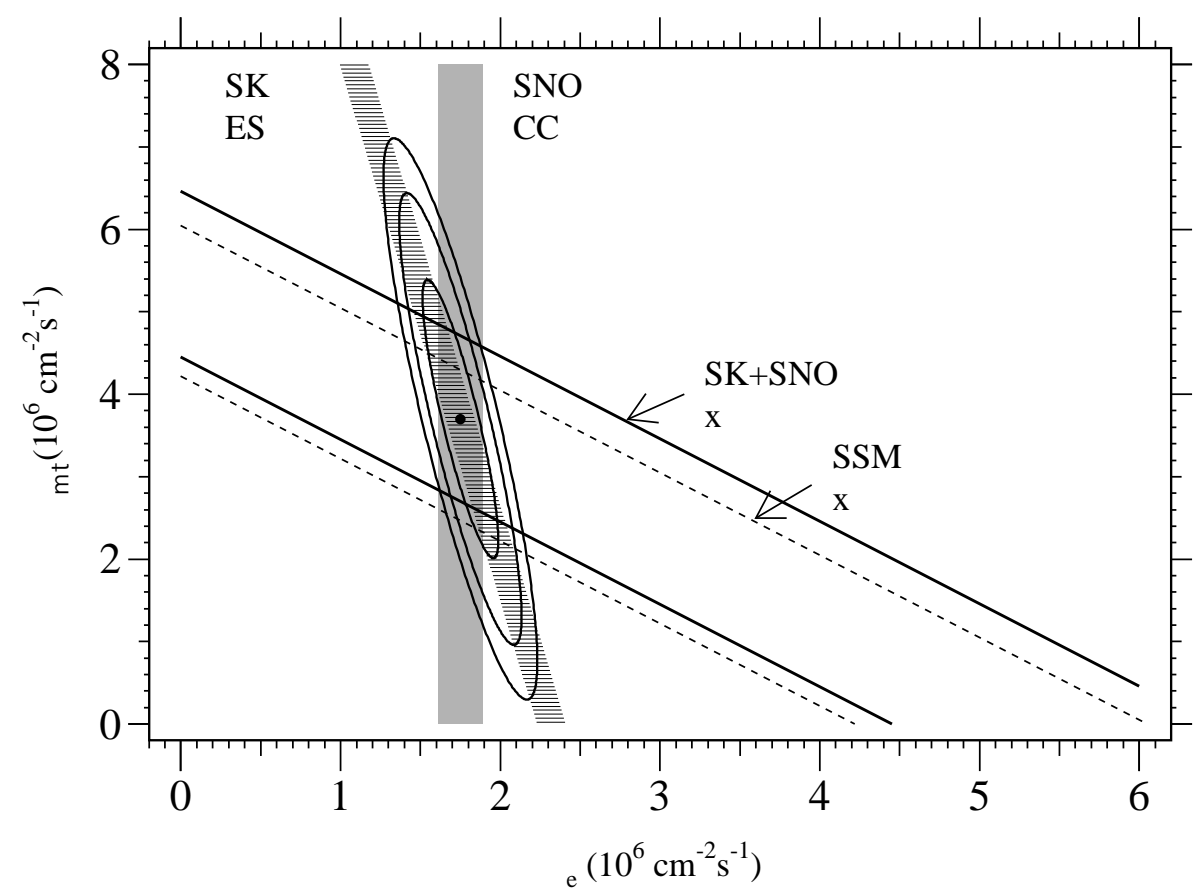

FIGURE 10. Flux of non-electron flavor active ${ }^{8} \mathrm{~B}$ solar neutrinos $\left(\phi\left(v_{\mu \tau}\right)\right)$ versus $\phi_{\mathrm{SNO}}^{\mathrm{CC}}$ and $\phi_{\mathrm{SK}}^{\mathrm{ES}}$. The band derived from the SNO and the Super-Kamiokande results $\left(\phi_{x}^{\mathrm{SK}+\mathrm{SNO}}\right)$ and the BPB01 prediction $\left(\phi_{x}^{\mathrm{SSM}}\right)$ are in good agreement.

\section{SUMMARY AND OUTLOOK}

Two significant results are reported in this paper. The data from SNO represent the first direct evidence that there is an active non-electron flavor neutrino component in the solar neutrino flux, and an exclusive oscillation from $v_{e}$ 's to sterile neutrinos is disfavored at the $3.1 \sigma$ level. This is also the first experimental determination of the total flux of active ${ }^{8} \mathrm{~B}$ solar neutrinos, which is in good agreement with the solar model predictions.

The SNO Collaboration is now analyzing the data from the pure $\mathrm{D}_{2} \mathrm{O}$ phase with a lowered energy threshold. Efforts are devoted to understanding the low energy $\beta \gamma$ and the photodisintegration contribution to the NC measurement. Results from this analysis will be reported in the near future.

The SNO experiment has just finished the first phase of the experiment. The deployment of $\mathrm{NaCl}$ to enhance the $\mathrm{NC}$ capability began on May 28, 2001. Figure 11 shows the detector background level seen in the Cherenkov data before, during and after the $\mathrm{NaCl}$ injection. The increase in the event rate during the injection is attributed to ${ }^{24} \mathrm{Na}$, which were activated by neutrons from the cavity wall when the $\mathrm{NaCl}$ brine was stored in the underground laboratory prior to the injection. After the injection has ended, one sees the decay of ${ }^{24} \mathrm{Na}$ with a characteristic $\tau_{1 / 2}=15$ hours. The background level in the detector returned to the pre-injection level after several days. After 8 months of running in this configuration, $\mathrm{SNO}$ will be able to make a definitive, solar model-independent statement (better than $6 \sigma$ ) on the solar neutrino oscillation hypothesis.

\section{ACKNOWLEDGEMENTS}

This research was supported by the Natural Sciences and Engineering Research Council of Canada, Industry Canada, National Research Council of Canada, Northern Ontario Heritage Fund Corporation and the Province of Ontario, the United States Department of Energy and in the United Kingdom by the Science and Engineering Research Council and the Particle Physics and Astronomy Research Council. Further support was provided by INCO, Ltd., Atomic Energy of Canada Limited (AECL), Agra-Monenco, Canatom, Canadian Microelectronics Corporation, AT\&T Microelectronics, Northern Telecom and British Nuclear Fuels, Ltd. The heavy water was loaned by AECL with the cooperation of Ontario Power Generation. 


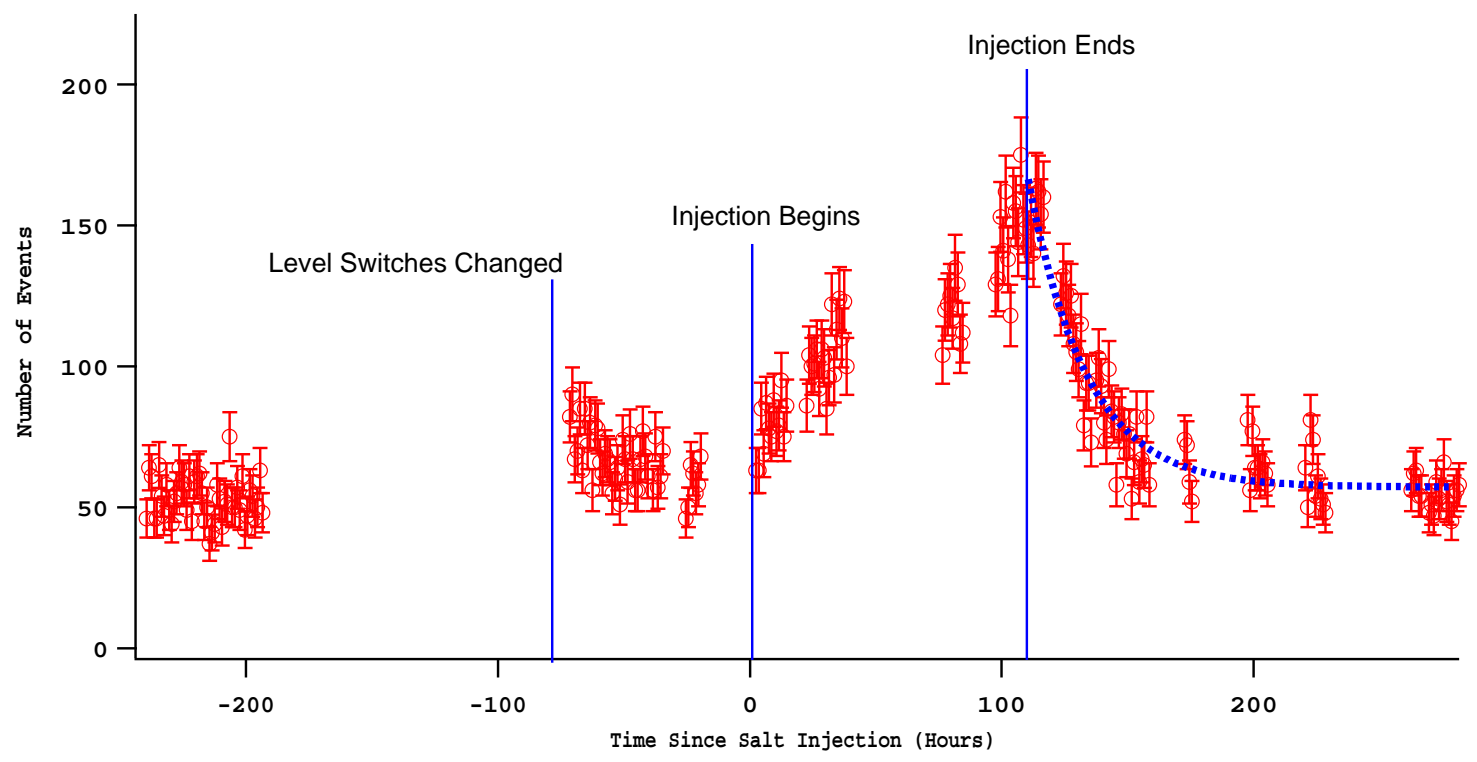

FIGURE 11. Event rate in a low energy background monitoring window before, during and after the $\mathrm{NaCl}$ injection. The gaps in this plot indicate detector down time, when detector hardware changes necessary to salt deployment were made. The dotted line is an exponential fit of the event rate. The fit is consistent with $\tau_{1 / 2}=15$ hours, which is the half life of ${ }^{24} \mathrm{Na}$.

\section{REFERENCES}

1. B. T. Cleveland et al., Astrophys. J. 496, 505 (1998).

2. K.S. Hirata et al., Phys. Rev. Lett. 65, 1297 (1990); K.S. Hirata et al., Phys. Rev. D44, 2241 (1991); K.S. Hirata et al., Phys. Rev. D45, 2170E (1992); Y. Fukuda et al., Phys. Rev. Lett. 77, 1683 (1996). This is the combined results from Kamiokande II and III.

3. J.N. Abdurashitov et al., Phys. Rev. C60, 055801 (1999). This is the most recent published result. The most recent unpublished result from the SAGE website (http://ewiserver.npl.washington.edu/SAGE/SAGE.html) is $77 \pm 6 \pm 3$ SNU, which include extractions up to December 2000.

4. W. Hampel et al., Phys. Lett. B447, 127 (1999). This is the combined results from GALLEX I, II, III, and IV.

5. M. Altmann et al., Phys. Lett. B490, 16 (2000).

6. S. Fukuda et al., Phys. Rev. Lett. 86, 5651 (2001).

7. J.N. Bahcall, M.H. Pinsonneault, and S. Basu, astro-ph/0010346 v2 (2001).

8. A.S. Brun, S. Turck-Chièze, and J.P. Zahn, Astrophys. J. 525, 1032 (1999); S. Turck-Chièze et al., Ap. J. Lett., 555 (2001).

9. The SNO Collaboration, Phys. Rev. Lett. 87, 071301 (2001).

10. The SNO Collaboration, Nucl. Instr. and Meth. A449, 172 (2000).

11. M.C. Browne et al., IEEE Trans. on Nucl. Sci. 46, 873 (1999).

12. M.R. Dragowsky et al., nucl-ex/0109011 (2001), accepted for publication in Nucl. Instr. and Meth. A.

13. The ${ }^{8}$ Li Calibration Source and Through-Going Muon Analysis in the Sudbury Neutrino Observatory, Ph.D. thesis, The University of Guelph (2001).

14. R.T. Ford, Nitrogen/Dye Laser System for the Optical Calibration of SNO, M.Sc. thesis, Queen's University (1993).

15. R.T. Ford, Calibration of SNO for Detection of ${ }^{8}$ B Neutrinos, Ph.D. thesis, Queen's University (1998).

16. B.A. Moffat, The Optical Calibration of the Sudbury Neutrino Observatory, Ph.D. thesis, Queen's University (2001).

17. A.W.P. Poon et al., Nucl. Instr. and Meth. A449, 172 (2000).

18. C.E. Ortiz et al., Phys. Rev. Lett. 85, 2909 (2000).

19. J.F. Beacom and P. Vogel, Phys. Rev. Lett. 83, 5222 (1999).

20. S. Nakamura, T. Sato, V. Gudkov and K. Kubodera, Phys. Rev. C63, 034617 (2001); M. Butler, J.-W. Chen, and X. Kong, Phys. Rev. C63, 035501 (2001); G. 't Hooft, Phys. Lett. 37B 195 (1971). The Butler et al. cross section with $L_{I, A}=5.6 \mathrm{fm}^{3}$ is used.

21. I.S. Towner, J. Beacom, and S. Parke, private communication; I.S. Towner, Phys. Rev. C58 1288 (1998); J. Beacom and S. Parke, hep-ph/0106128; J.N. Bahcall, M. Kamionkowski and A. Sirlin, Phys. Rev. D51 6146 (1995).

22. G.L. Fogli, E. Lisi, A. Palazzo, and F.L. Villante, Phys. Rev. D63, 113016 (2001); F.L. Villante, G. Fiorentini and E. Lisi, Phys. Rev. D59, 013006 (1999); G.L. Fogli, E. Lisi, D. Montanino, and A. Palazzo, hep-ph/0106247 (2001). The most recent paper in this series indicates that the Super-Kamiokande threshold should be $8.6 \mathrm{MeV}$, instead of $8.5 \mathrm{MeV}$ that is used here. 